\title{
Curing and thermal behavior of epoxy resins of hexafluoro - bisphenol -A and bisphenol-A
}

\author{
Vaishnav Kiran ${ }^{1}$ and Bharti Gaur ${ }^{1 *}$ \\ ${ }^{1}$ Research and Development Laboratory, Department of Chemistry, National Institute of Technology \\ Hamirpur, Himachal Pradesh, India \\ *bhartigaur@gmail.com
}

\begin{abstract}
This paper describes the synthesis and characterization of epoxy resins based on (hexafluoroisopropylidene)diphenol (EFN) and p,p'-isopropylidenebisphenol (EBN), respectively and 4, 4'- (hexafluoroisopropylidene)dipthalic-imideamine (IMAM), a curing agent. The synthesized epoxy resins and IMAM curing agent were characterized by Fourier Transform Infrared (FTIR) and ${ }^{1} \mathrm{H}$ Nuclear Magnetic Resonance (NMR) spectroscopy. ${ }^{13} \mathrm{C}$ NMR technique was also used to characterize IMAM. Study of curing behavior of EFN and EBN with stoichiometric amount of aromatic 4,4'-diaminodiphenylmethane (DDM), 4,4'-diaminodiphenylsulfone (DDS) and IMAM by using Differential Scanning Calorimetery (DSC) indicated that IMAM was least reactive curing agent towards both epoxy resins as compared to DDS and DDM. The investigation of thermal decomposition of the cured compounds by thermogravimetric analyzer (TGA) indicated the higher thermal stability of EFN and EBN resins initially with DDS and at elevated temperatures with IMAM. It was also observed that EFN resins were thermally more stable than EBN resins cured with corresponding curing agents.
\end{abstract}

Keywords: aromatic diamines, curing behavior, epoxy resins, imide amine, thermal properties.

\section{Introduction}

Epoxy resins are one of the most important thermosetting resins known to possess good mechanical properties, high chemical resistance and excellent adhesive properties. These desirable properties make them widely applicable materials viz for surface coatings, adhesives, corrosion protectants, composites and laminates, encapsulants for semiconductors, insulating materials for electronic devices etc $^{[1-10]}$. However the conventional epoxy resins do not exhibit very high thermal resistance, which is a prerequisite for advanced materials. Over the years multifunctional epoxy resins have been synthesized by many researchers to improve the thermal stability of these resins, either by incorporating aromatic ring as in case of phenolic based resins or introducing phosphorus in the epoxy backbone ${ }^{[11-13]}$. Attempts have also been made to introduce fluorinated substituents into the polymer backbone in order to improve electrical properties such as dielectric constant. These fluorinated substituents are also reported to reduce the moisture absorption due to the nonpolar character of fluorocarbons ${ }^{[14]}$. Some typical structures of multifunctional epoxy resin are shown in Figure 1. These resins are reported to be used as matrix material for high performance fiber-reinforced composites in the aerospace industry and as encapsulant for electronic components.

Outstanding properties and performance of epoxy resins are obtained by crosslinking these into a three-dimensional, insoluble and infusible network by reacting with suitable curing agents. The crosslinking occurs through reaction of the epoxide or oxirane group. In the recent years attention has also been focused by the researchers on the development of novel curing agents that will help to improve the performance of epoxy resins at elevated temperatures. This article reports the introduction of pendant fluoro groups on the epoxy backbone in order to increase the thermal stability of the epoxy resin. An attempt has also been made to host these fluorinated substituents as well as the aromatic rings on the backbone of the curing agent, an Imide-amine, in order to obtain matrix which could show even better heat stability. Curing and decomposition behavior of fluorine containing epoxy resin (EFN) was compared with bisphenol-A novolac based (EBN) epoxy resin by using aromatic DDM, DDS and IMAM as curing agents.

\section{Materials and Methods}

\subsection{Materials}

4,4'-diaminodiphenlymethane, 4,4'-diaminodiphenyl sulfone, p,p'-isopropylidenebisphenol (bisphenol-A/BPA) and 4,4'- (hexafluoroisopropylidene) dipthalicanhydride were purchased from Alfa Aesar. Formaldehyde, tetrahydrofuran (THF) and glacial acetic acid were received from Fisher Scientific, Epichlorohydrin (ECH) and sodium hydroxide were obtained from Loba Chemie. Isopropanol and methyl isobutyl ketone (MIBK) were purchased from Merck, 4,4'-(hexafluoroisopropylidene)diphenol (hexafluoro/6Fbisphenol-A/6F-BPA) was obtained from Sigma Aldrich and paratoluene sulfonic acid (PTSA) from laboratory reagents, sodium bicarbonate was of analytical grade.

\subsection{Synthesis and Characterization}

2.2.1 Synthesis of 4, 4'-(hexafluoro-isopropylidene) - diphenol (6F-BPA) and p,p'-isopropylidenebisphenol (BPA) based novolac resins (Scheme 1 and 2)

A 40ml methyl isobutyl ketone (MIBK) solution containing 6F-BPA $(0.05 \mathrm{~mol})$ and PTSA $(0.0014 \mathrm{~mol})$ was added to a $250 \mathrm{ml}$ three- necked round bottom flask equipped with 


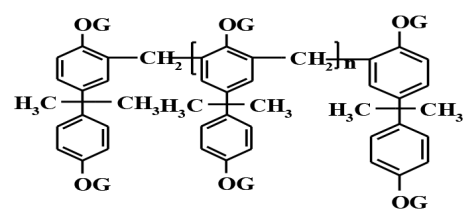

Bisphenol novolac epoxy resin<smiles></smiles>

PhoPhosphorous containing tetraglycidyl epoxy resin

OG<smiles>COC1CO1</smiles>

G<smiles>C[C@H]1CO1</smiles>

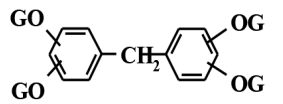

Resorcenol formaldehyde type epoxy resin (F-76)<smiles>CC(C)c1ccc(Cc2ccc(N(C)C)cc2)cc1</smiles>

Tetraglycidyl diamine diphenolmethane<smiles>O=C(O)C1COCC1C(=O)O</smiles>

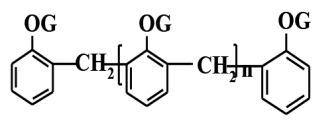

Novolac epoxy resin

Cycloaliphatic epoxy resin

4-Diglycidylether of benzotrifluoride

Figure 1. Some typical structures of multifunctional epoxy resins ${ }^{[1-13]}$.<smiles>FC(F)(F)c1ccc(OC(COC2CO2)C(OC2CO2)C2CO2)cc1</smiles>

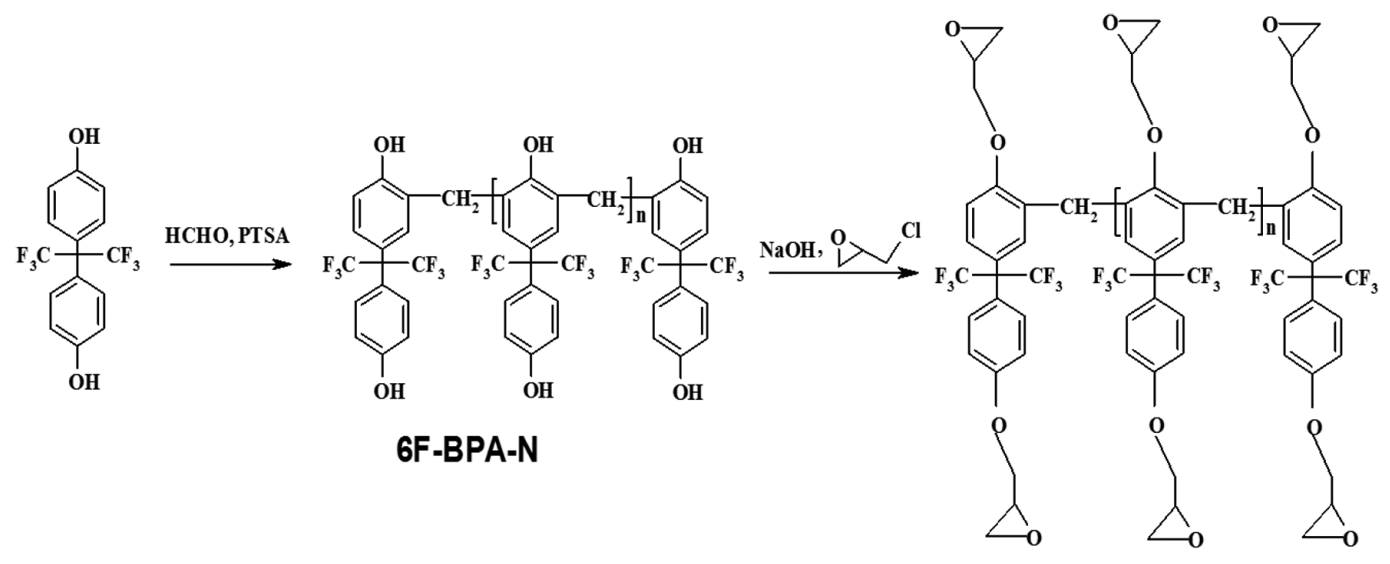

EFN

Scheme 1. Synthesis of hexafluorobisphenol-A novolac epoxy resin (EFN).

mechanical stirrer and dean and stark trap with a reflux condenser. Nitrogen gas was purged for 30 minutes and the reaction solution was heated to $100^{\circ} \mathrm{C}$ with stirring. $0.08 \mathrm{~mol}$ of formaldehyde solution (37-41\%) was added to the reaction mixture drop wise. Then, the reaction mixture was heated to $120^{\circ} \mathrm{C}$ with constant stirring and maintained at this temperature for five hours. The condensation of novolac resin is a reversible process; therefore $(0.13 \mathrm{~mol})$ of water generated during the reaction was removed as azeotropic mixture with MIBK. The reaction mixture was cooled to room temperature and washed with deionised water several times until it became neutral. The solution was then distilled at $120^{\circ} \mathrm{C}$ in order to remove the MIBK solvent. The product was then washed with a mixture of water/methanol $(2: 1 \mathrm{v} / \mathrm{v})$ 
several times to remove the unreacted $6 \mathrm{~F}-\mathrm{BPA}$. A red brown solid product was obtained after vacuum drying at $60^{\circ} \mathrm{C}$ for $48 \mathrm{~h}$.Similar procedure was followed for the synthesis of BPA based novolac resin.

2.2.2 Synthesis of novolac epoxy resins of 4, 4'-(hexafluoroisopropylidene) - diphenol (EFN) and p,p'- isopropylidenebisphenol (EBN) (Scheme 1 and 2)

To the above dried product of 6F-BPA novolac $8 \mathrm{~mol}$ of epichlorohydrin for every phenolic group of novolac resin and isopropyl alcohol $(0.83 \mathrm{~mol})$ was added to $250 \mathrm{ml}$ three necked round bottom flask equipped with mechanical stirrer and condenser. After increasing the reaction temperature to $70^{\circ} \mathrm{C}$ with constant stirring, $0.078 \mathrm{~mol}$ of $20 \mathrm{wt} \%$ aqueous solution of sodium hydroxide was added drop wise into the reaction solution within one hour. The system was maintained at $70^{\circ} \mathrm{C}$ for another four hours with constant stirring. The reaction product in the flask was washed several times with deionized water to remove the sodium chloride. The product was dissolved in toluene and filtered in order to remove residual salt. Toluene and excess of ECH were distilled off under reduced pressure. Finally the product obtained was dried at $60-70^{\circ} \mathrm{C}$ under vacuum for 48 hours. BPA based novolac epoxy resin (EBN) was also synthesized by adopting a similar procedure.

2.2.3 Synthesis of 4, 4'- (hexafluoroisopropylidene)diphthalicimideamine (IMAM) curing agent (Scheme 3)

$0.011 \mathrm{~mol}$ of DDM was dissolved in $40 \mathrm{ml}$ glacial acetic acid in a round bottom flask equipped with condenser. To this $0.005 \mathrm{~mol}$ of 4,4 '- (hexafluoroisopropylidene)diphthalic anhydride was added. The reaction mixture was heated at $120^{\circ} \mathrm{C}$ for ten hours with constant stirring. The prepared 4, 4'-(hexafluoroisopropylidene)diphthalic imideamine (IMAM) was precipitated in ice-cold water, filtered and washed several times with distilled water followed by washings with sodium bicarbonate solution. The product was again washed with distilled water several times before drying it in vacuum oven at $50-60^{\circ} \mathrm{C}$.

\subsection{Characterization}

\subsubsection{Structural characterization}

FTIR spectra of the samples were recorded by using Perkin Elmer 1600 FTIR spectrophotometer in the range of $4000-400 \mathrm{~cm}^{-1}$ on the $\mathrm{KBr}$ pellets. ${ }^{1} \mathrm{H}$ NMR and ${ }^{13} \mathrm{C}$ NMR spectra were recorded on a BRUKER AVANCE II 400 NMR spectrometer using deuterated Dimethylsulfoxide as solvent, and tetramethylsilane as the internal standard.

\subsubsection{Curing and decomposition behavior}

The samples for curing studies were freshly prepared at $25^{\circ} \mathrm{C}$ by mixing the novolac epoxy resins EFN and EBN with three different curing agents DDM/DDS/IMAM, dissolved in appropriate solvents respectively, in the molar ratio of $1: 2$ in small glass vial with vigorous stirring using a glass rod until the samples became homogeneous in nature. DDM and DDS were dissolved in minimum amount of acetone whereas IMAM in minimum amount of THF. The solvents were then removed by vacuum stripping before recording the DSC scans. DSC scans for all these samples under dynamic conditions were obtained on Mettler Differential Scaning Calorimetery with programmed heating rate of $10^{\circ} \mathrm{C} \mathrm{min}^{-1}$

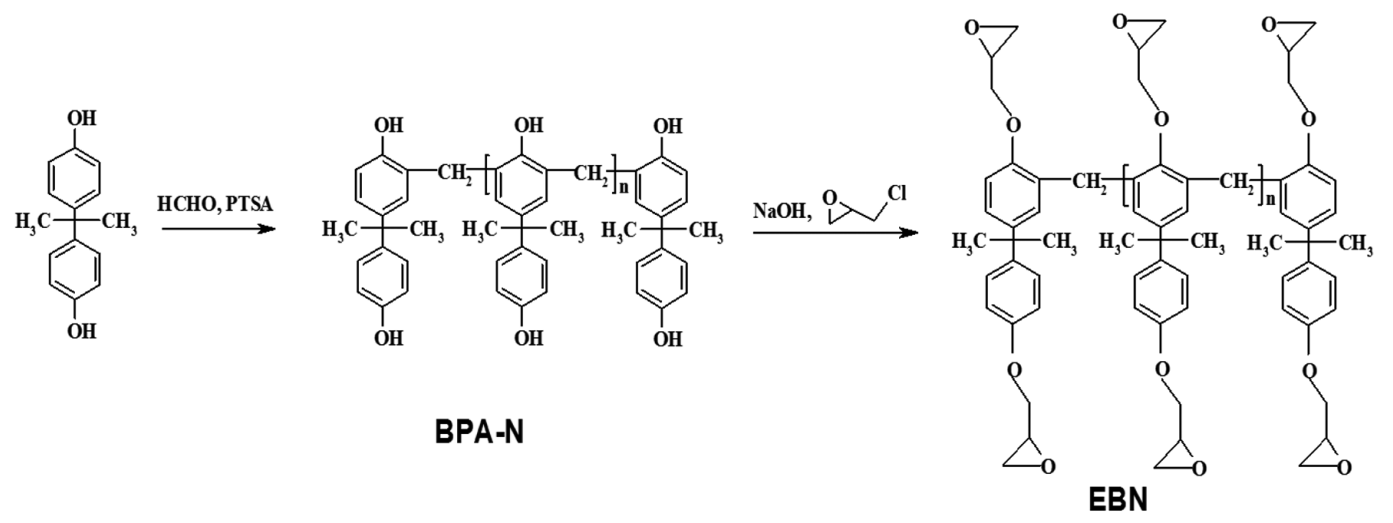

Scheme 2. Synthesis of bisphenol-A novolac epoxy resin (EBN).

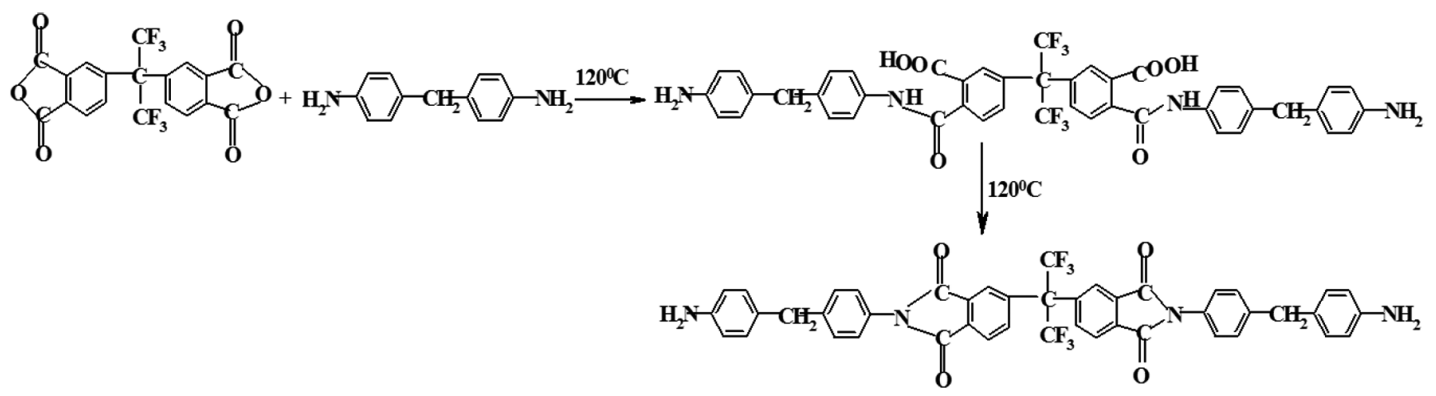

Scheme 3. Synthesis of 4, 4'- (hexafluoroisopropylidene)diphthalic-imideamine (IMAM). 
from $35^{\circ} \mathrm{C}$ to the temperature at which exothermic reactions were completed.

Thermal stability of the samples cured isothermally at $250 \pm 10^{\circ} \mathrm{C}$ in hot air oven was evaluated by thermogravimetry in nitrogen atmosphere (flow rate $=200 \mathrm{ml} / \mathrm{min}$ ). EXSTAR TG/DTA 6300 was used to record TG/DTG/DTA traces at heating rate of $10^{\circ} \mathrm{C} \mathrm{m^{-1 }}$ with sample size of $10 \pm 1 \mathrm{mg}$. The relative thermal stabilities of the different cured resins were quantitatively estimated by comparing the temperature at which maximum degree of weight loss occurred and by determining the activation energy of the thermal decomposition reaction.

\subsubsection{Epoxide equivqlent weight}

Epoxide equivalent weights (EEWs) of synthesized epoxy resins EFN and EBN were determined by the pyridinium chloride method ${ }^{[15]}$ were found to be 354 and 377 respectively.

\section{Results and Discussions}

\subsection{Characterization of 6F-BPA and BPA novolacs and their epoxy resins}

The FTIR spectra of 6F-Bisphenol-A novolac (6F-BPA-N) (Figure 2a) and Bisphenol-A novolac (BPA-N) resins showed the $-\mathrm{CH}_{2}$ - stretching vibration due to bridging

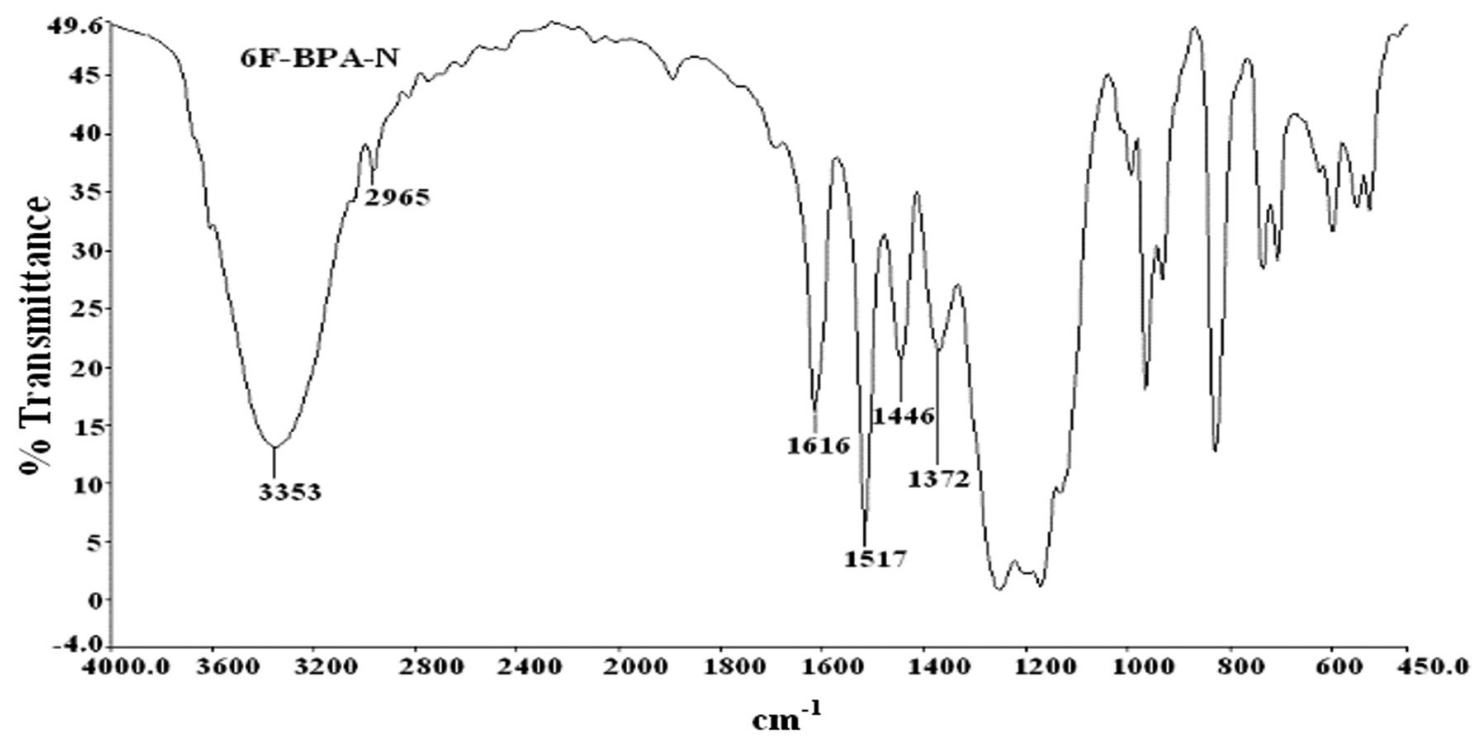

(a)

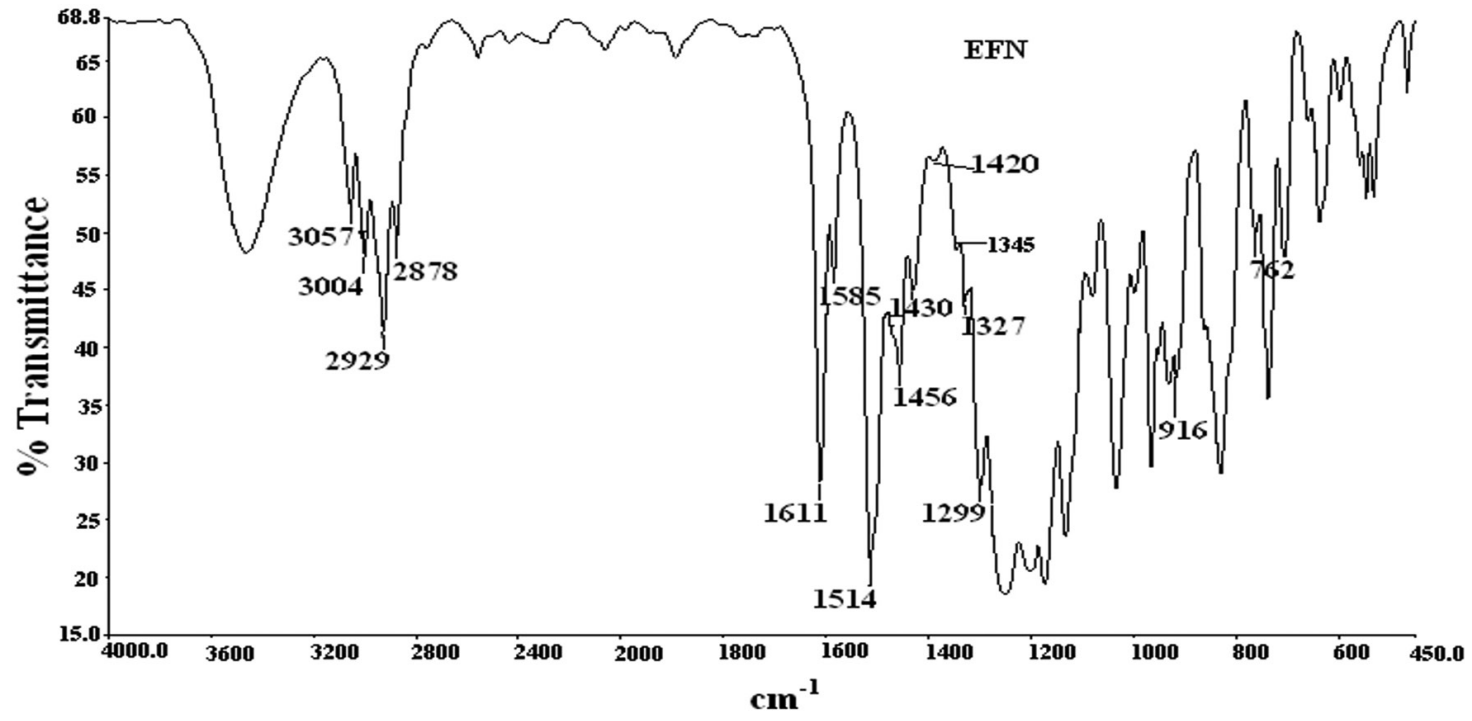

(b)

Figure 2. FTIR spectra of (a) 6F-BPA novolac; (b) EFN novolac epoxy resin. 
methyene groups at $2965 \mathrm{~cm}^{-1}$. A broad band was observed at $3353 \mathrm{~cm}^{-1}$ due to the hydroxyl group. Apart from the other peaks appearing for the aromatic $\mathrm{C}-\mathrm{H}$ stretching, $\mathrm{C}=\mathrm{C}$ stretching, $-\mathrm{CH}_{2}-$ bending, as in case of BPA-N resin. IR spectrum of $6 \mathrm{~F}-\mathrm{BPA}-\mathrm{N}$ resin showed peaks in the region of $1372-1446 \mathrm{~cm}^{-1}$, which indicated the presence of C-F bonds.

The FTIR spectra of EFN (Figure 2b) and EBN novolac epoxy resins showed characteristic absorptions at 3057 and $2929 \mathrm{~cm}^{-1}$ due to streching vibrations of aromatic rings and bridging methylene groups, respectively. A characteristic absorption band at $1299 \mathrm{~cm}^{-1}$ depicted the ring breathing frequency of epoxy ring, the appearance of band at $916 \mathrm{~cm}^{-1}$ proved the asymmetric ring deformation and band at $762 \mathrm{~cm}^{-1}$ showed the symmetric ring deformation of epoxy ring. The spectrum of EFN (Figure 2b) also showed peaks in between $1372-1446 \mathrm{~cm}^{-1}$ due to the presence C-F bonds.

Figure 3 shows the ${ }^{1} \mathrm{H}$ NMR spectra of $6 \mathrm{~F}-\mathrm{BPA}-\mathrm{N}$, BPA-N novolac resins and EFN, EBN novolac epoxy resins. Figures $3 \mathrm{a}$ and $\mathrm{c}$ showed characteristic proton resonance signal at 3.7 ppm due to methylene $\left(-\mathrm{CH}_{2}\right)$ bridging protons and multiplet for aromatic protons at 6.6-7.1 ppm. A singlet due to $-\mathrm{OH}$ protons of phenol appeared at $9.5 \mathrm{ppm}$ in the spectrum of 6F-BPA-N (Figure $3 \mathrm{a}$ ) and at $8.7 \mathrm{ppm}$ in the spectrum of BPA-N (Figure 3c). BPA-N resin also showed a characteristic singlet due to $-\mathrm{CH}_{3}$ protons at $1.5 \mathrm{ppm}$ (Figure 3c). ${ }^{1} \mathrm{HNMR}$ spectra (Figures $3 \mathrm{~b}$ and $3 \mathrm{~d}$ ) of EFN and EBN showed proton resonance signals at 3.6-3.8 and 6.7-7.3 ppm due to $-\mathrm{CH}_{2}$ bridging and aromatic protons respectively. Characteristic proton resonance signals at
2.7-2.9, 3.3 and 3.9-4.2 ppm due to $-\mathrm{O}-\mathrm{CH}_{2},-\mathrm{CH}$ and $-\mathrm{CH}_{2}$ protons of epoxy ring were also observed, respectively.

\subsubsection{Characterization of IMAM curing agent}

The FTIR spectrum of IMAM curing agent in Figure $4 \mathrm{a}$ showed characteristic absorption bands at 1783 and $1725 \mathrm{~cm}^{-1}$ due to asymmetric and symmetric stretching vibration of imide group and a band was observed at $722 \mathrm{~cm}^{-1}$ due to five-membered ring deformation of the cyclic imide. $-\mathrm{NH}_{2}$ stretching bands appeared at $3260 \pm 30 \mathrm{~cm}^{-1}$. Absorption bands for $\mathrm{C}-\mathrm{N}$ and $\mathrm{C}=\mathrm{C}$ stretching of aromatic rings were observed at $1409-1435 \mathrm{~cm}^{-1}$ and $1602-1672 \mathrm{~cm}^{-1}$, respectively. ${ }^{1} \mathrm{H}$ NMR spectrum Figure $4 \mathrm{~b}$ of IMAM curing agent showed proton resonance signal at $3.9 \mathrm{ppm}$ for $-\mathrm{NH}_{2}$ protons and 7.0-8.1 ppm for aromatic protons. A resonance signal appeared at $2.0 \mathrm{ppm}$ due to methylene protons. ${ }^{13} \mathrm{C}$ NMR spectrum (Figure $4 \mathrm{c}$ ) was recorded in order to confirm the cyclization of the amic acid which is an intermediate in the reaction represented by the Scheme 3. The characteristic signal at $168 \mathrm{ppm}$ due to cyclic imide carbon was observed. Carbons of $\mathrm{Ar}-\mathrm{CH}_{2}$ and Ar- $\mathrm{NH}_{2}$ appeared at 132 and 141 ppm respectively. Peaks due to aromatic carbons were observed at 119-137 ppm.

\subsection{Thermal curing behavior}

The curing behavior of EFN and EBN novolac epoxy resins with three different curing agents were studied by DSC. The reactivity of the curing agents towards epoxy resins can be examined from the onset exothermic temperature. The onset exothermic temperature was obtained by extrapolating the steepest portion of the curve to the base line. A curing agent

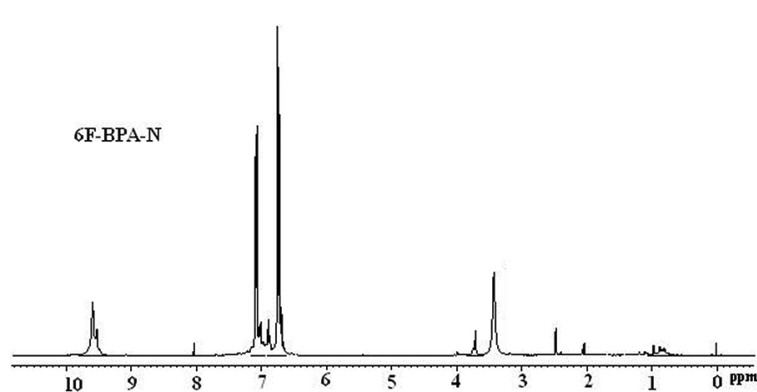

(a)

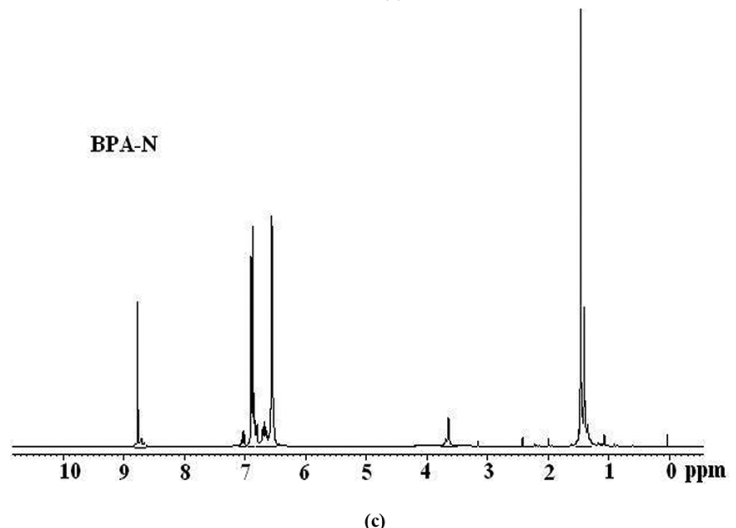

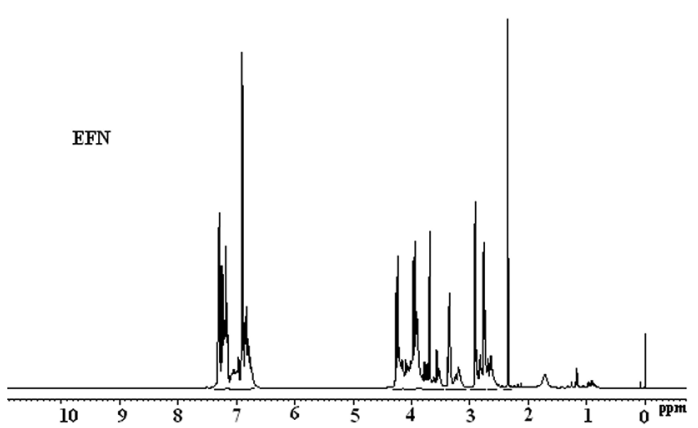

(b)

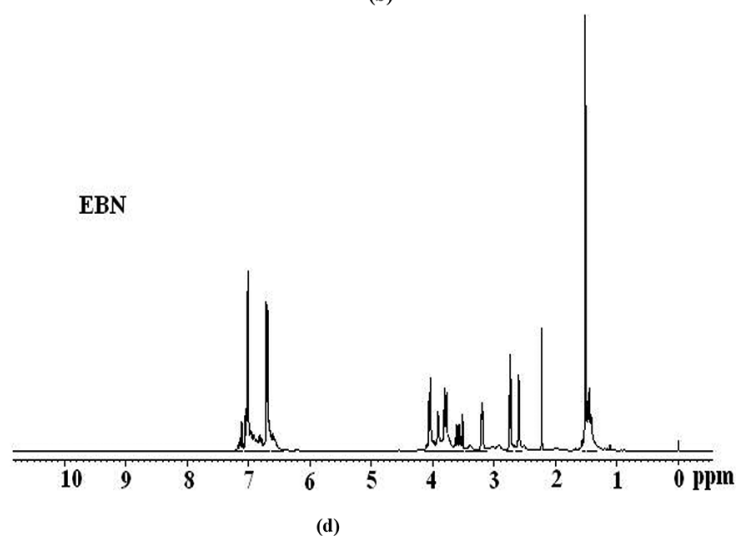

Figure 3. 'HNMR of (a) 6F-BPA novolac; (b) EFN novolac epoxy resin; (c) BPA novolac; (d) EBN novolac epoxy resin. 
that exhibits lower onset exothermic temperature is more reactive towards the epoxy resin. Figures 5 and 6 show the typical DSC scans for curing of EFN and EBN epoxies with DDS and IMAM curing agents respectively. The exothermic temperatures, onset, peak and endset temperatures have been given in Table 1 . The enthalpy change $(\Delta H)$ which can be used to determine the extent of curing of $\operatorname{resin}^{[16,17]}$ have been calculated by measuring the area under the exothermic transition and are also summarized in Table 1. The onset curing exothermic temperature of EFN with DDM/DDS/IMAM were observed at $118,184,240^{\circ} \mathrm{C}$, respectively. Curing exotherms of EBN with DDM/DDS/ IMAM showed the onset curing temperatures at 126, 147, $256^{\circ} \mathrm{C}$ respectively. The reaction of the amine with an oxirane ring is a nucleophilic addition reaction. From the results of thermal curing it was observed that despite the presence of

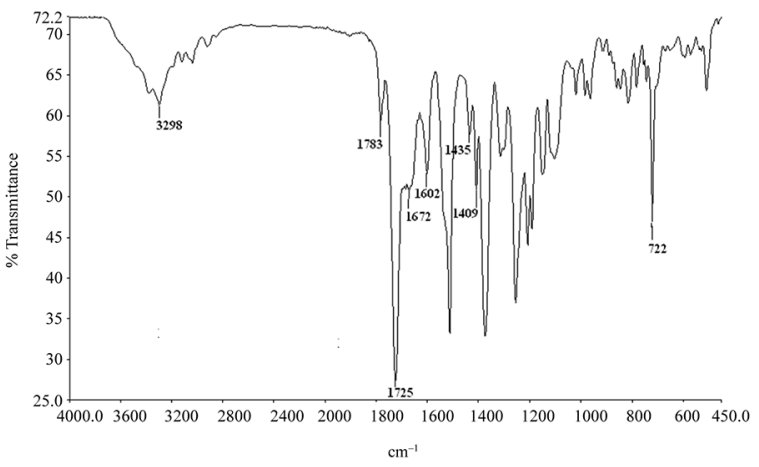

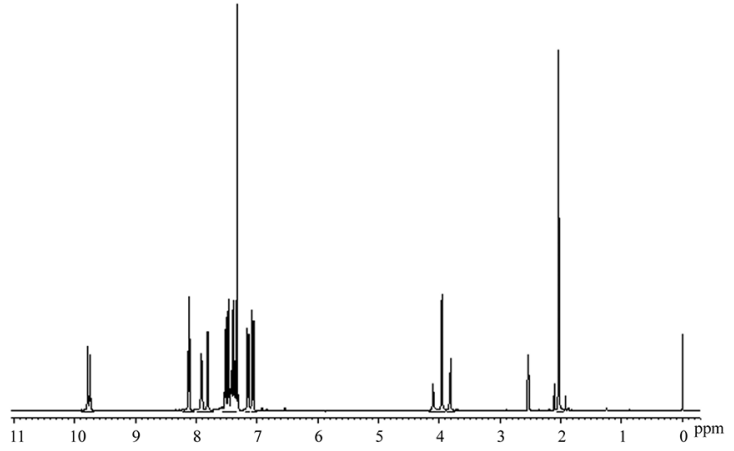

(b)

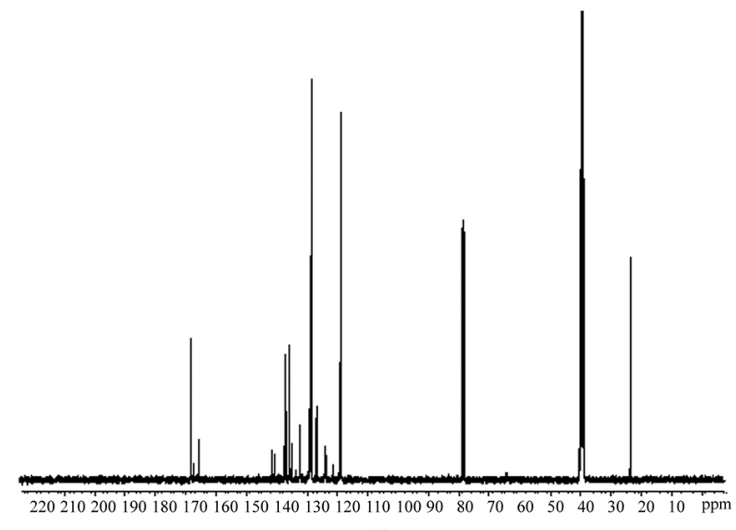

(c)

Figure 4. (a) FTIR; (b) ${ }^{1} \mathrm{H}$ NMR of IMAM curing agent; (c) ${ }^{13} \mathrm{C}$ NMR of IMAM curing agent.

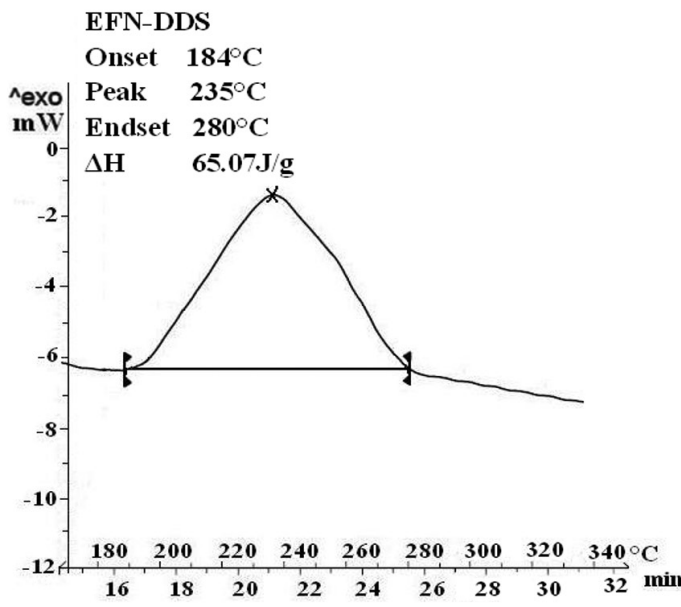

(a)

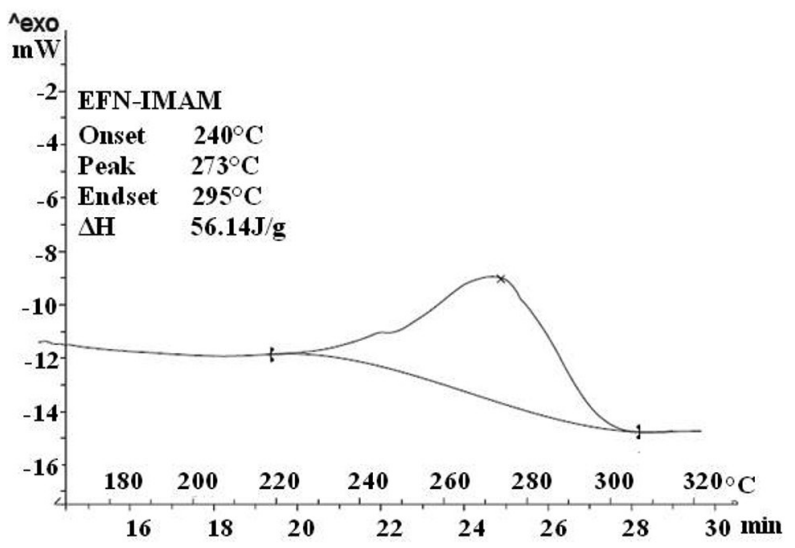

(b)

Figure 5. DSC scans of novolac based epoxy resins of (a) EFN/DDS; (b) EFN/IMAM. 


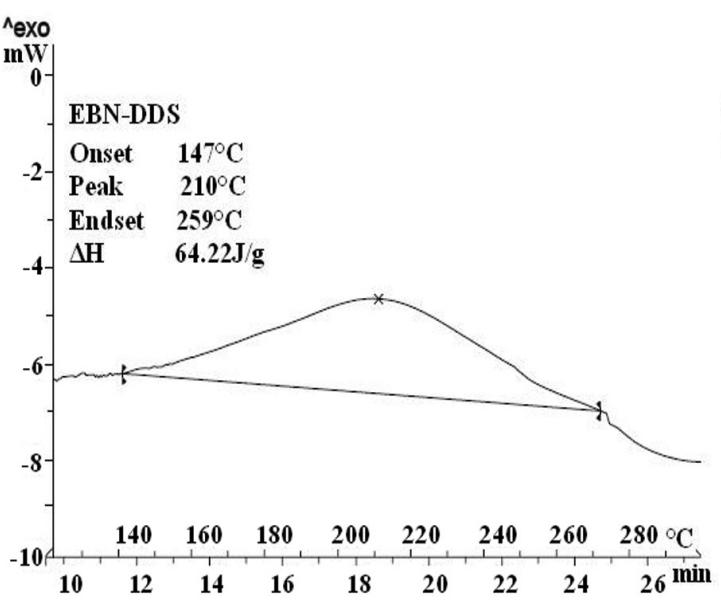

(a)

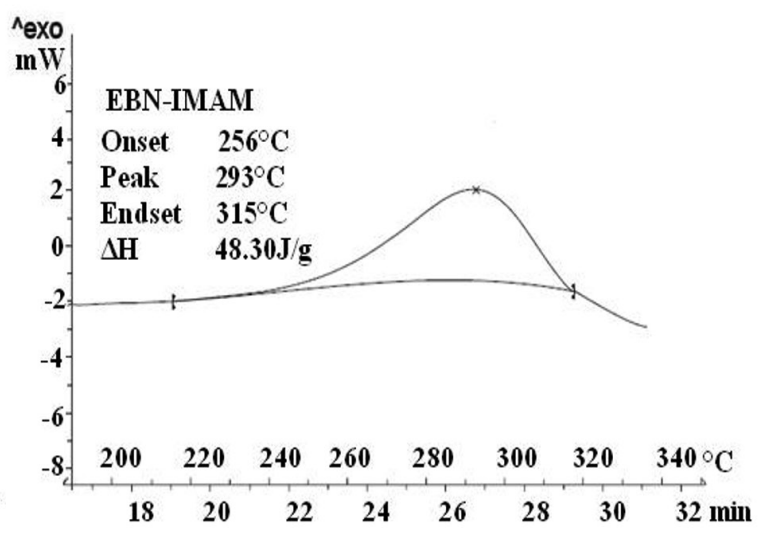

(b)

Figure 6. DSC scans of novolac based epoxy resins of (a) EBN/DDS; (b) EBN/IMAM.

Table 1. DSC results of cured epoxy resins.

\begin{tabular}{ccccc}
\hline Sample Designation & $\mathbf{T}_{\text {onset }}\left({ }^{\circ} \mathbf{C}\right)$ & $\mathbf{T}_{\text {peak }}\left({ }^{\circ} \mathbf{C}\right)$ & $\mathbf{T}_{\text {endset }}\left({ }^{\circ} \mathbf{C}\right)$ & $\Delta \mathbf{H}(\mathbf{J} / \mathbf{g})$ \\
\hline EFN- DDM & 118 & 144 & 194 & 99.81 \\
EFN- DDS & 184 & 235 & 280 & 65.07 \\
EFN-IMAM & 240 & 273 & 295 & 56.14 \\
EBN- DDM & 126 & 134 & 154 & 73.88 \\
EBN- DDS & 147 & 210 & 259 & 64.22 \\
EBN- IMAM & 256 & 293 & 315 & 48.30 \\
\hline
\end{tabular}

electron donating methyl group in IMAM it was found to be the least reactive. The reason for this may be attributed to the presence of electron withdrawing $\mathrm{CF}_{3}$ and carbonyl groups in the main chain as well as the steric hinderance caused due to the bulkiness of the groups. This reduced the basicity of amine and consequently the reactivity of the IMAM curing agent decreased. Whereas in DDS only electronic factor dominated and owing to the electron withdrawing nature of sulfone group the reactivity of DDS was found to be higher than IMAM. The highest reactivity of DDM towards both the epoxy resins was in good agreement with electronic donating effects of methane moiety ${ }^{[1,10,18]}$. Thus the order of basicity of amine groups in the three curing agents will be DDM $>$ DDS $>$ IMAM. Furthermore, the melting point of DDS $\left(175^{\circ} \mathrm{C}\right)$ and $\operatorname{IMAM}\left(195^{\circ} \mathrm{C}\right)$ is very high as compared to $\mathrm{DDM}\left(90^{\circ} \mathrm{C}\right)$, which may also have a negative effect on their reactivity. The EFN epoxy resin was observed to be more reactive towards the curing agents as compared to that EBN which may be probably due to electronic factors. The presence of electron withdrawing groups $\mathrm{CF}_{3}$ may facilitate the oxirane ring opening during the reaction with the amine group.

\subsection{Thermal properties of cured resins}

EFN and EBN epoxy resin samples were isothermally cured at $250 \pm 10^{\circ} \mathrm{C}$ for twelve hours with stoichiometric amount of diamines or imide-amine curing agents. DSC scans were recorded for these samples and absence of any residual exothermic peak in their scans confirmed complete curing of the samples. TGA technique considered to be the most favorable technique for rapid estimation in comparing and ranking the thermal stability of samples, was employed for determining the thermal stability of these isothermally cured samples. The relative thermal stability of the cured samples was compared by observing the initial degradation temperatures (IDT), temperature of maximum degradation $\left(\mathrm{T}_{\max }\right)$, final degradation temperature (FDT) and residual weight retention $\left(\mathrm{R}_{\mathrm{w}}\right)$ at $900^{\circ} \mathrm{C}$. The results have been summarized in Table 2 .

The TG curves of cured epoxy resins EFN/DDM, EFN/DDS, EFN/IMAM are shown in Figure 7. It can be seen that the samples did not show initial weight loss till $345^{\circ} \mathrm{C}$ which implied that the cured samples of EFN resin were thermally stable till this temperature. As the temperature reached $350 \pm 5^{\circ} \mathrm{C}$ these samples showed a slight weight loss and it was observed that the EFN/DDS sample showed better thermal stability as compared to EFN/DDM and was found to be thermally less stable than that of EFN/IMAM. However as the scanning temperature reached $415 \pm 5^{\circ} \mathrm{C}$ rapid thermal degradation occurred and EFN/DDS found to be less stable as compared to corresponding EFN/DDM and EFN/IMAM respectively and the final decomposition temperatures of all the samples were found to be almost identical.

Figure 8a shows typical TG/DTG/DTA scans of cured epoxy resin of EBN with IMAM and Figure $8 \mathrm{~b}$ shows the TG curves for cured EBN/DDM, EBN/DDS and EBN/IMAM, respectively. From the Figure $8 \mathrm{~b}$ and Table 2 it can be observed that the cured EBN resins containing DDM/DDS/IMAM, respectively, were thermally stable till the scanning temperature reached up to $330 \pm 5^{\circ} \mathrm{C}$. As the 
Table 2. Thermal properties of cured epoxy resins.

\begin{tabular}{ccccccc}
\hline Sample Designation & IDT $\left({ }^{\circ} \mathbf{C}\right)$ & $\mathbf{T}_{\max }\left({ }^{\circ} \mathbf{C}\right)$ & FDT $\left({ }^{\circ} \mathbf{C}\right)$ & $\begin{array}{c}\text { Residual weight } \\
\text { retention }\left(\mathbf{R}_{w}\right) \%\end{array}$ & $\begin{array}{c}\text { Activation energy of decomposition } \\
\text { reaction }\left(\mathbf{E}_{\mathbf{a}}\right)\end{array}$ \\
\hline EFN-DDM & & & & & $\begin{array}{c}\text { Temperature } \\
\text { Range }\left({ }^{\circ} \mathbf{C}\right)\end{array}$ & $\mathbf{E}_{\mathbf{a}}(\mathbf{K J} / \mathbf{m o l})$ \\
EFN-DDS & 354 & 422 & 540 & 26.46 & $290-690$ & 62.825 \\
EFN-IMAM & 359 & 417 & 542 & 22.48 & $290-625$ & 48.742 \\
EBN-DDM & 369 & 456 & 544 & 29.08 & $280-670$ & 74.208 \\
EBN-DDS & 339 & 409 & 465 & 22.83 & $265-560$ & 63.836 \\
EBN-IMAM & 350 & 407 & 470 & 17.65 & $280-560$ & 59.773 \\
\hline
\end{tabular}

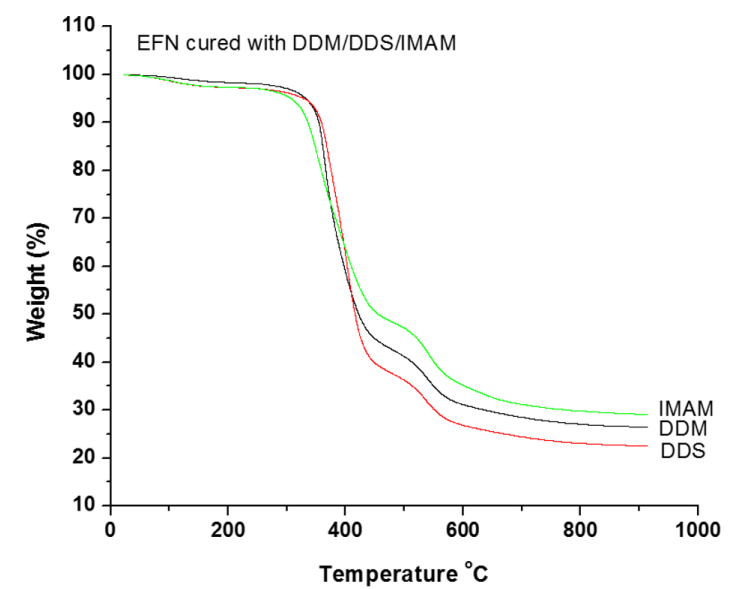

Figure 7. TG curves for EFN cured with DDM/DDS/IMAM.

scanning temperature rose above $340^{\circ} \mathrm{C}$, the cured EBN/DDS sample showed the values of IDT, Tmax, FDT as 350, 407, and $470^{\circ} \mathrm{C}$ respectively. However the FDT for the sample EBN/IMAM was found to be higher as compared EBN cured with DDM and DDS respectively.

From the values of IDT and FDT it was observed that initially better thermal stability of EFN and EBN resins with DDS may be due to the rigidity provided by high functionality and rigid structure of DDS as compared to that cured with $\mathrm{DDM}^{[19]}$. For the superior thermal stability of EFN and EBN resins cured with IMAM, It is assumed that the solid state aromatization reaction in the case of IMAM are promoted by the formation of the crosslinks which may develop between the carbonyl and trapped or unreacted amino groups at elevated temperatures and thus may have increased the thermal stability of the sample. It was also seen that the cured EFN and EBN resins with IMAM showed highest residual weight retentions $\left(\mathrm{R}_{\mathrm{w}}\right)$ at $900^{\circ} \mathrm{C}$. This was probably due to the presence of thermally oxidative resistant $\mathrm{CF}_{3}$ groups in the main chain of IMAM. Furthermore the cured EFN resins were found to be thermally more stable as compared to cured EBN resins. The enhanced thermal properties of cured EFN samples were probably due to the presence of fluorinated groups in the EFN network structure.

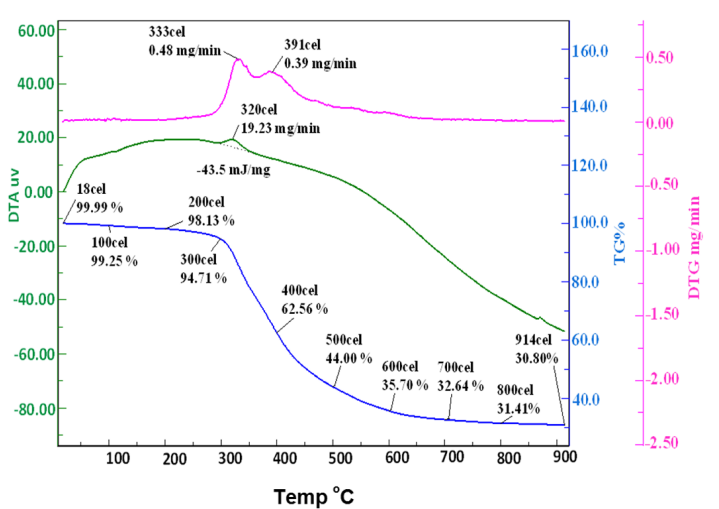

(a)

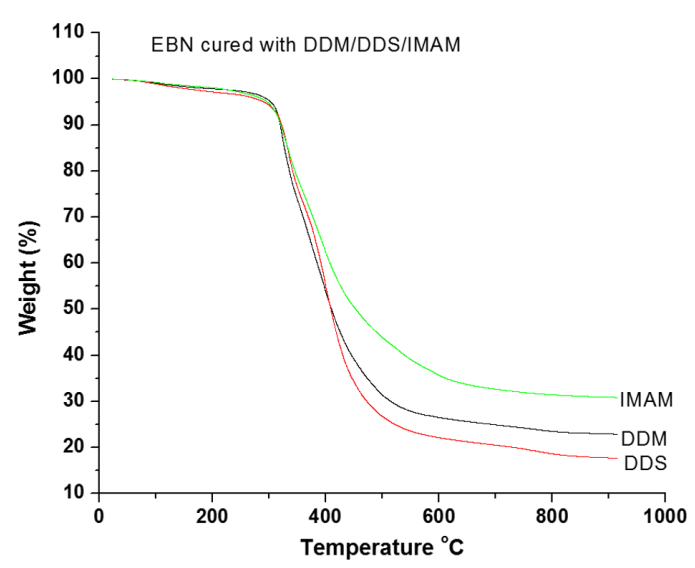

(b)

Figure 8. (a) Typical TG/DTG/DTA scans for cured sample of EBN/IMAM; (b) TG curves for EBN cured with DDM/DDS/ IMAM.

\subsubsection{Activation energy of decomposition $\left(E_{a}\right)$}

Activation energy of decomposition $\left(\mathrm{E}_{\mathrm{a}}\right)$ can also be used as a decisive factor for evaluating the thermal stability of the polymers, higher the value of activation energy higher will be the stability of the compounds. Because the rate constant of reaction depends upon energy difference between initial and transistion states of the reaction. If higher is the value of the energy difference higher will be the activation energy and more will be the stability of the compound. Calculation of activation energy from the TG curves has 
been reported in the literature by several methods ${ }^{[20-24]}$. But the expression used by Dharwadkar and Kharkhanawala for the calculation of activation energy is independent of sample size and heating rate.

$$
\ln \left[\ln (1-\alpha)^{-1}\right]=\frac{E_{a} 100 \theta}{R T_{i}^{2}\left(T_{f}-T_{i}\right)}+C
$$

where $\alpha$, fraction reacted; $T_{i}$, the temperature of inception of reaction; $T_{f}$, temperature at the point of inflection on the thermogram; $\theta=\left(\mathrm{T}-\mathrm{T}_{\mathrm{s}}\right.$ ) (where $\mathrm{T}$ is the temperature under consideration and $\mathrm{T}_{\mathrm{s}}$ is the maximum temperature); $\mathrm{C}$, constant; R, gas constant. A plot of $\ln \left[\ln (1-\alpha)^{-1}\right]$ versus $\theta$ gave a straight line was equal to

$$
m=\frac{100 E_{a}}{R T_{I}^{2}\left(T_{f}-T_{i}\right)}
$$

From this equation, $\mathrm{E}_{\mathrm{a}}$ was calculated.

Activation energies of decomposition $\left(\mathrm{E}_{\mathrm{a}}\right)$ were calculated by using Equations 1 and 2 and the values of $\mathrm{E}$ have been summarized in Table 2. On comparing the activation energies of decomposition reaction of EFN and EBN novolac epoxy resins cured with DDM/ DDS/ IMAM curing agents respectively, the following trend was observed for activation energies [(EFN / EBN $)+$ IMAM $]>$ $[(\mathrm{EFN} / \mathrm{EBN})+\mathrm{DDM}]>[(\mathrm{EFN} / \mathrm{EBN})+\mathrm{DDS}]$.

These results also substantiate the fact that the presence of fluorinated groups in the curing agent IMAM have also contributed towards the improvement of thermal stability of both the cured epoxy resins. From Table 2 it was also observed that the cured samples having high Rw value showed higher activation energy which led to the increase in their thermal stability.

\section{Conclusions}

EFN and EBN novolac epoxy resins were synthesized, which were then thermally cured with aromatic amines (DDM, DDS) and synthesized aromatic imide-amine (IMAM) curing agents. The investigation of curing behavior of cured epoxy resins revealed that IMAM was found to be the least reactive curing agent towards both EFN and EBN as compared to their counterparts DDM and DDS. This was attributed to the electronic effects, high molecular weight and physical properties of IMAM curing agent. The experimental results for the thermal degradation demonstrated that the major weight loss for all the samples occurred in the temperature range of $300-600^{\circ} \mathrm{C}$. Both the EFN and EBN samples cured with IMAM exhibited higher thermal stability probably due to the solid state aromatization reactions which may lead to the formation of cross-links between the carbonyl and trapped or unreacted amino groups. The presence of $\mathrm{CF}_{3}$ groups in IMAM may also have played its role in the enhancement of thermal stability of such cured resins. The EFN cured resins were found to be more heat resistant as compared to their corresponding EBN resins. This was owing to the ease of oxirane ring opening due to the presence of $\mathrm{CF}_{3}$ groups in EFN that might have been a good ignition for the formation of better crosslinked network structure than EBN cured resins.

\section{References}

1. Lee, G. S., Lee, Y. C., \& Gong, M. S. (2001). Prepration of epoxy resins containing ether ether sulfone unit and thermal properties. Bulletin of Korean Chemical Society, 22(12), 13931396.

2. Romao, B. M. V., Diniz, M. F., Azevedo, M. F. P., Lourenco, V. L., Pardini, L. C., Dutra, R. C. L., \& Burel, F. (2006). Characterization of the curing agents used in epoxy resins with TG/FT-IR technique. Polimeros: Ciencia e Technologia, 16(2), 94-98. http://dx.doi.org/10.1590/S0104-14282006000200007.

3. Paluvai, N. R., Mohanty, S., \& Nayak, S. K. (2014). Synthesis and modification of epoxy resins and their composites: A review. Polymer-Plastics Technology and Engineering, 53(16), 17231758. http://dx.doi.org/10.1080/03602559.2014.919658.

4. Tao, Z., Yang, S., Ge, Z., Chen, J., \& Fan, L. (2007). Synthesis and properties of novel fluorinated epoxy resins based on 1,1-bis(4-glycidyllesterphenyl)-1-(3'-trifuoromethylphenyl)2,2,2-trifluoroethane. European Polymer Journal, 43(2), 550-560. http://dx.doi.org/10.1016/j.eurpolymj.2006.10.030.

5. Vinicius, P., Bluma, S. G., \& Raquel, M. S. (2013). Influence of the polyhedral oligomeric silsesquioxane n-phenylaminopropyposs in the thermal stability and the glass transition temperature of epoxy resin. Polímeros: Ciencia e Technologia, 23(3), 331-338. http://dx.doi.org/10.4322/polimeros.2013.039.

6. Alhousami, M. H. M., Al-Kamali, A. S. N., \& Athawale, A. A. (2014). Synthesis and characterization of novel sulphanilamide/ epoxy resin modified polyester for thermal stability and impact strength. Open Journal of Polymer Chemistry, 4(4), 115-127. http://dx.doi.org/10.4236/ojpchem.2014.44013.

7. Dixit, V., Nagpal, A. K., \& Singhal, R. (2010). Synthesis and characterization of phenoxy modified epoxy blends. Malaysian Polymer Journal, 5(2), 69-83. Retrieved in 20 December 2014, from http://www.fkkksa.utm.my/mpj

8. Oliveira, A., Backer, C. M., \& Amico, S. C. (2015). Evaluation of the characteristics of an epoxy resin with diffrent degassing agents. Polímeros: Ciência e Tecnologia, 25(2), 186-191. http:// dx.doi.org/10.1590/0104-1428.1661

9. Yu, M., Feng, B., Xie, W., Fang, L., Li, H., Liu, L., Ren, M., Sun, J., Zhang, J., \& Hu, H. (2015). The modification of a terafunctional epoxy and its curing reaction. Materials, $8(6)$, 3671-3684. http://dx.doi.org/10.3390/ma8063671.

10. Ge, Z., Tao, Z., LiuJ., Fan, J., \& Yang, S. (2007). Synthesis and charaterization of novel trifunctional fluorine containing epoxy resins based on 1,1,1-Tris(2,3-epoxypropoxyphenyl)2,2,2-trifluoroethane. Polymer Journal, 39(11), 1135-1142. http://dx.doi.org/ 10.1295/polymj.PJ2007096.

11. Cheng, J., Li, J., \& Zhang, J. Y. (2009). Curing behavior and thermal properties of trifunctional epoxy resin cured by 4 , 4'-diaminodiphenyl sulfone. Express Polymer Letters, 3(8), 501-509. http://dx.doi.org/10.3144/expresspolymlett.2009.62.

12. Heo, G. Y., \& Park, S. J. (2009). Effect of substituted trifluoromethyl groups on thermal and mechanical properties of fluorine containing epoxy resin. Macromolecular Research, 17(11), 870-873. http://dx.doi.org/10.1007/BF03218628.

13. Meenakshi, K. S., Pradeep, E., Sudhan, J., \& Kumar, S. A. (2012). Development and characterization of new phosphorus based flame retardant tetraglycidyl epoxy nanocomposites for aerospace application. Bulletin of Materials Science, 35(2), 129-136. http://dx.doi.org/10.1007/s12034-012-0271-0.

14. Liu, J. G., He, M. H., Li, Z. X., Qian, Z. G., Wang, F. S., \& Yang, S. Y. (2002). Synthesis and characterization of organosoluble polyimides with trifluoro-substituted benzene in the side chain. 
Journal of Polymer Science. Part A, Polymer Chemistry, 40(10), 1572-1582. http://dx.doi.org/10.1002/pola.10240.

15. Knoll, D. W., Nelson, D. H. \& Keheres, P. W. (1958). Paint, plastics and printing ink chemistry. In 134th American Chemical Society Meeting (Paper No. 5, pp. 20). Chicago: Division of Paint, Plastics and Printing Ink Chemistry.

16. Maka, H., \& Spychaj, T. (2012). Epoxy resin crosslinked with conventional and deep eutectic ionic liquids. Polimery, 57(6), 456-462. http://dx.doi.org/10.14314/polimery.2012.456.

17. Gaceva, G. B., \& Buzarovska, A. (2013). A rapid method for the evaluation of cure kinetics of thermosetting polymers. Macedonian Journal of Chemistry and Chemical Engineering, 32(2), 337-344. Retrieved in 20 December 2014, from http:// www. researchgate.net/260245399

18. Costa, L. M., Pardini, C. L., \& Rezende, C. M. (2005). Influence of aromatic amine hardness in the cure kinetics of an epoxy resin used in advanced composites. Materials Research, 8(1), 65-70. http://dx.doi.org/10.1590/S1516-14392005000100012.

19. Su, W. F., Huang, H. W., \& Pan, P. W. (2002). Thermal properties of rigid rod epoxies cured with diaminodiphenyl sulfone and dicyandiamide. Thermochimica Acta, 392/393, 391-394. http:// dx.doi.org/10.1016/S0040-6031(02)00125-9.

20. Freeman, E. S., \& Carroll, B. (1958). The application of thermoanalytical techniques to reaction kinetics: the thermogravimetric evaluation of the kinetics of the decomposition of calcium oxalate monohydrate. Journal of Physical Chemistry, 62(4), 394-397. http://dx.doi.org/10.1021/j150562a003.

21. Coats, A. W., \& Redfern, J. (1964). Kinetic parameters from thermogravimetric data. Nature, 201(4914), 68-69. http:// dx.doi.org/10.1038/201068a0.

22. Horowitz, H. H., \& Metzger, G. (1963). A new analysis of thermogravimetric traces. Analytical Chemistry, 35(10), 14641468. http://dx.doi.org/10.1021/ac60203a013.

23. Dharwadkar, S. R., Kharkhanawala, M. D., Schwenker, R. P., \& Garn, P. D. (1969). Thermal analysis in organic materials and physical chemistry. New York: Academic Press.

24. Tripathi, G., \& Srivastava, D. (2011). Study on the effect of carboxyl terminated butadiene acrylonitrile $(\mathrm{CTBN})$ copolymer concentration on the decomposition kinetics parameters of blends of glycidyl epoxy and non-glycidyl epoxy resin. International Journal of Organic Chemistry, 1(3), 105-112. http://dx.doi.org/10.4236/ijoc.2011.13016.

Received: Dec. 20, 2014

Revised: Aug. 15, 2015

Accepted: Aug. 17, 2015 